\title{
Fractional quantum transport and staggered topological transition in a lossy trimerized lattice
}

\author{
Lei Du, ${ }^{1,2}$ Jin-Hui Wu, ${ }^{1, *}$ M. Artoni, ${ }^{3, \dagger}$ and G. C. La Rocca ${ }^{4, \ddagger}$ \\ ${ }^{1}$ Center for Quantum Sciences and School of Physics, Northeast Normal University, Changchun 130117, China \\ ${ }^{2}$ Beijing Computational Science Research Center, Beijing 100193, China \\ ${ }^{3}$ Department of Engineering and Information Technology and INO-CNR Sensor Laboratory, Brescia University, \\ 25133 Brescia, Italy \\ ${ }^{4}$ Scuola Normale Superiore and CNISM, 56126 Pisa, Italy
}

(Received 6 August 2019; published 4 November 2019)

\begin{abstract}
Transport in a non-Hermitian trimerized lattice with one lossy site per unit cell exhibits a quantized mean displacement before decay characterized by a two-step phase transition from zero to unity through a fractional value. Such a peculiar behavior, sensitive to both the lattice parameters and the initial state, is shown to be closely related to the presence of two nondegenerate dark states decoupled from the lossy sites and correspond to two staggered band closing points. We further check that distinct topological phases can also be characterized by the total Zak phase and by the number of midgap edge states. Moreover, we demonstrate that the edge states are topologically protected and their localization can be controlled via the couplings imbalance. Our results are expected to be relevant for implementations of topological photonics such as those based on arrays of evanescently coupled waveguides.
\end{abstract}

DOI: 10.1103/PhysRevA.100.052102

\section{INTRODUCTION}

Topological insulators (TIs) are a new phase of quantum matter and can exhibit robust electron transport through protected surface or edge states [1,2]. While Hermitian TIs have been well explored and understood, non-Hermitian TIs are drawing more and more interest due to their unique topological properties associated with the exceptional points (EPs) [3-14]. This then inspires extensive studies on topological photonic systems, where inhomogeneous gain and loss are ubiquitous and easily controlled [15-18]. Many breakthroughs have been achieved in such systems with robust optical delay lines [19], backscattering-free edge states [20], topological polaritons [21], and topological lasing [22-24] being a few paradigms. As two most prototypical models allowing for nontrivial topological phases, the Su-Schrieffer-Heeger (SSH) [25-34] and Aubry-André-Harper (AAH) [35-37] models have been widely used for non-Hermitian extensions by introducing various dissipative effects. Relevant Hamiltonians typically contain complex on-site potentials [38-41] but may also exhibit non-Hermitian hopping rates [42-45]. Both of the two cases allow the realization of exotic topological phenomena with no Hermitian counterparts, such as anomalous edge states [8], non-Bloch bulk-boundary correspondence [45], and non-Hermitian localization transitions [42].

The winding number related to the Zak phase [46-48] and protected by specific symmetries [49-51] is usually adopted to characterize the topological features of one-dimensional (1D) TIs. It is always an integer in Hermitian systems with the

\footnotetext{
*jhwu@nenu.edu.cn

†artoni@lens.unifi.it

†larocca@sns.it
}

$0( \pm 1)$ value(s) presenting the topological trivial (nontrivial) phase(s). It can be half integers, however, in non-Hermitian systems in the presence of two nondegenerate EPs $[8,52]$. It may also take integers of absolute values larger than unity, yielding thus richer phase diagrams in some extended topological models with long-range hopping terms [53,54]. A feasible way for observing the winding number via the mean displacement of a particle was originally formulated in a pioneering work that relied on a non-Hermitian SSH model [25]. It is shown that a 1D tight-binding lattice with two sites per unit cell (the first one being lossy and the second one not) is characterized by the ratio between intracell $(v)$ and intercell $\left(v^{\prime}\right)$ hopping terms. Initializing a particle on the nonlossy site at the origin, the mean displacement $\langle\Delta m\rangle$ at which the particle decays during the time evolution is studied: when $v>v^{\prime}$ it is found that $\langle\Delta m\rangle=0$ (i.e., the particle on average does not move before decaying) and when $v<v^{\prime}$ instead $\langle\Delta m\rangle=1$ (i.e., the particle on average decays in the next unit cell). While this result is expected in the two limiting cases $v \gg v^{\prime}$ (the particle cannot leave the initial unit cell) and $v \ll v^{\prime}$ (the particle has to move to the next unit cell to find a lossy site), it is striking that the value of $\langle\Delta m\rangle$ is quantized and the transition at $v=v^{\prime}$ is sharp. As a matter of fact, the mean displacement does correspond to a topological winding number [25], which is connected to the Zak phase $[28,46]$. This ground-breaking result has been experimentally observed [27] and theoretically generalized to Hermitian [55] and non-Hermitian models [28,56-58]. Recent experiments have also reported the winding number measurement in Floquet systems by examining the photonic quantum walk $[59,60]$.

Here we investigate a non-Hermitian trimerized lattice containing one lossy site and two neutral (i.e., nondecaying) sites in each unit cell and three distinct nearest-neighbor 


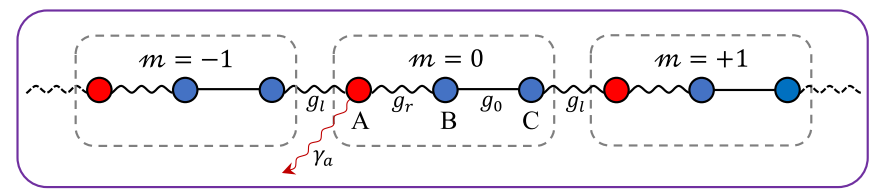

FIG. 1. Diagram of the non-Hermitian trimerized lattice under consideration. Each unit cell contains one lossy site $A_{m}$ with decay rate $\gamma_{a}$ as well as two neutral sites $B_{m}$ and $C_{m}$, which are coupled via the intercell $\left(g_{l}\right)$ and intracell $\left(g_{r}\right.$ and $\left.g_{0}\right)$ coupling constants.

couplings. As it lacks chiral and inversion symmetry, this model is qualitatively different from those most commonly studied dimerized lattices such as the SSH model. Our model is also different from other trimerized lattices with, e.g., neither gain nor loss [61], balanced [62] or unbalanced [63] gain and loss. It has been chosen to be a nearest-neighbor lattice with only one lossy site per unit cell, conditions under which its quantum transport properties are expected to be topologically protected [28]. Here, the mean displacement before decay as a function of coupling imbalance exhibits a two-step phase transition between the trivial phase with $\langle\Delta m\rangle=0$ and the nontrivial phase with $\langle\Delta m\rangle=1$ through an intermediate phase with fractional $\langle\Delta m\rangle$, depending on both the on-site potentials and initial state choice. At variance with the dimerized models such as the SSH one [25] exhibiting a one-step transition from $\langle\Delta m\rangle=0$ to $\langle\Delta m\rangle=1$, this peculiar two-step transition is associated with two different dark states [25,28] decoupled from all lossy sites, and may also be characterized by a two-step transition of the total Zak phase of values 0,1 , and 2 (in units of $2 \pi$ ). The two transition points correspond precisely to two staggered closing points in the upper and lower band gaps, which separate the three phases with different numbers of topologically protected midgap states. Besides a thorough investigation of the trimerized case, we also extend our analysis to the case of a tetramerized lattice showing a three-step topological transition.

\section{MODELS AND TOPOLOGICAL INVARIANTS}

\section{A. Non-Hermitian trimerized lattice}

We start by considering in Fig. 1 a $1 \mathrm{D}$ lossy trimerized lattice containing $2 N+1$ unit cells of indices $m \in$ $\{-N, \ldots,-1,0,1, \ldots, N\}$. Each unit cell consists of one lossy site $\left(A_{m}\right)$ and two neutral sites $\left(B_{m}\right.$ and $\left.C_{m}\right)$ from left to right. The intercell coupling constant between sites $C_{m-1}$ and $A_{m}$ is denoted by $g_{l}$ while the intracell coupling constant between sites $A_{m}\left(B_{m}\right)$ and $B_{m}\left(C_{m}\right)$ is denoted by $g_{r}\left(g_{0}\right)$. Then the real-space Hamiltonian of our model can be written as

$$
\begin{aligned}
H(m)= & -i \gamma_{a}|m, a\rangle\left\langle m, a\left|+\delta_{b}\right| m, b\right\rangle\langle m, b| \\
& +\delta_{c}|m, c\rangle\langle m, c|+\left[g_{l}|m, a\rangle\langle m-1, c|\right. \\
& \left.+g_{r}|m, b\rangle\left\langle m, a\left|+g_{0}\right| m, c\right\rangle\langle m, b|+\text { H.c. }\right]
\end{aligned}
$$

with $\gamma_{a}$ being the decay rate of the lossy sites $A_{m}$ while $\delta_{b}$ and $\delta_{c}$ are the on-site potentials (with respect to the energy of the lossy site) of the neutral sites $B_{m}$ and $C_{m}$, respectively. Setting

$$
|\psi(t)\rangle=\sum_{m}\left[a_{m}(t)|m, a\rangle+b_{m}(t)|m, b\rangle+c_{m}(t)|m, c\rangle\right]
$$

as a general state of this lattice, it is straightforward to attain from Eq. (1) the following dynamic equations

$$
\begin{aligned}
\frac{\partial a_{m}}{\partial t} & =-\gamma_{a} a_{m}-i g_{l} c_{m-1}-i g_{r} b_{m}, \\
\frac{\partial b_{m}}{\partial t} & =i \delta_{b} b_{m}-i g_{r} a_{m}-i g_{0} c_{m}, \\
\frac{\partial c_{m}}{\partial t} & =i \delta_{c} c_{m}-i g_{0} b_{m}-i g_{l} a_{m+1} .
\end{aligned}
$$

Without loss of generality, we assume in the following $\delta_{b}=$ $-\delta_{c}=\delta_{0}$ and

$$
g_{0}=1, \quad g_{l}=1+\delta g, \quad g_{r}=1-\delta g,
$$

with $\delta g$ being the coupling imbalance of the trimerized lattice.

The use of non-Hermitian Hamiltonians is the simplest way to take losses into account. A more rigorous treatment of an open quantum system would require a Lindblad masterequation approach including quantum jumps; the latter treatment, however, can be shown in some regimes of experimental interest to be reasonably well approximated by non-Hermitian Hamiltonians which do neglect quantum jumps $[29,64]$.

It is worth mentioning that recent experiments on topological photonics based on arrays of evanescently coupled waveguides implement the framework above: in the paraxial approximation Maxwell's wave equation is turned into a Schrödinger-like equation where the role of the time coordinate $t$ is played by the propagation distance $z$ along the waveguides [17,27].

\section{B. Mean displacement}

Supposing the initial state is $|\psi(0)\rangle=b_{0}|0, b\rangle+c_{0}|0, c\rangle$, which is a superposition of the two central neutral sites and constrained by $\left|b_{0}\right|^{2}+\left|c_{0}\right|^{2}=1$, we can define the mean displacement of a particle before its decay [25,27]

$$
\langle\Delta m\rangle=2 \gamma_{a} \int_{0}^{\infty} d t\left(\sum_{m} m\left|a_{m}(t)\right|^{2}\right)
$$

as a topological invariant to examine the topological phase diagram via numerical simulations.

First we focus on two specific initial states characterized, respectively, by $\left|\psi_{1}(0)\right\rangle=|0, b\rangle\left(b_{0}=1\right)$ and $\left|\psi_{2}(0)\right\rangle=$ $|0, c\rangle\left(c_{0}=1\right)$ for a finite sample of $2 N+1=31$ unit cells. Figure 2 shows that a single topological phase transition from $\langle\Delta m\rangle=0$ (trivial) to $\langle\Delta m\rangle=1$ (nontrivial) occurs around $\delta g=0$ in the case of $\delta_{0}=0$. The phase transition splits, however, into two steps in the case of $\delta_{0} \neq 0$ with a fractional $\langle\Delta m\rangle$ appearing as the intermediate phase. It is worth noting that as $\delta_{0}$ is gradually increased, the fractional $\langle\Delta m\rangle$ exists on the one hand within a more and more extended region identical for both initial states of our interest, but on the other hand exhibits a smaller and smaller (larger and larger) value for the initial state $\left|\psi_{1}(0)\right\rangle=|0, b\rangle\left(\left|\psi_{2}(0)\right\rangle=|0, c\rangle\right)$. We finally note that upon initializing the particle on the lossy site $\left(\left|\psi_{3}(0)\right\rangle=|0, a\rangle\right)$, the mean displacement always vanishes $(\langle\Delta m\rangle=0)$, similar to the case of the non-Hermitian SSH model [25]. 

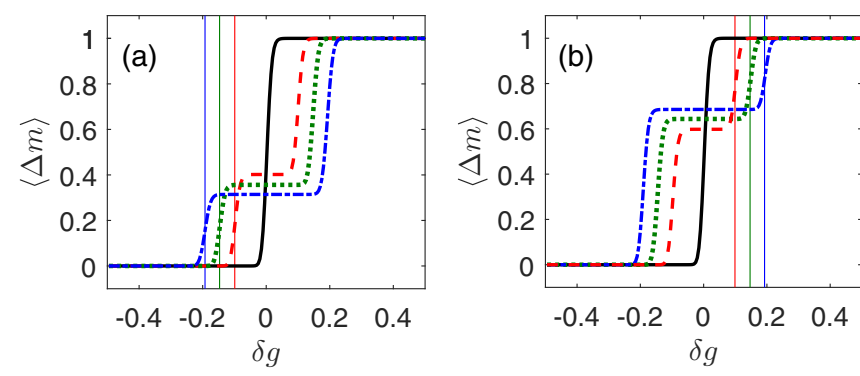

FIG. 2. Mean displacement $\langle\Delta m\rangle$ against coupling imbalance $\delta g$ with the initial state (a) $\left|\psi_{1}(0)\right\rangle=|0, b\rangle$ and (b) $\left|\psi_{2}(0)\right\rangle=|0, c\rangle$. The black solid, red dashed, green dotted, and blue dot-dashed lines correspond to $\delta_{0}=0, \delta_{0}=0.2, \delta_{0}=0.3$, and $\delta_{0}=0.4$, respectively. Other parameters are chosen as $\gamma_{a}=0.2$ and $N=15$.

\section{Dark states}

Considering that with periodic boundary conditions the system is translationally invariant, it may be beneficial to understand the above results in the momentum representation by performing the Fourier transformation $O_{m}=\sum_{k} O_{k} e^{i m k}$ $(O=a, b, c)$. In this way, we can attain the following bulk eigenequation:

$$
H(k)\left|\psi_{k}\right\rangle=E(k)\left|\psi_{k}\right\rangle,
$$

with $\left|\psi_{k}\right\rangle=a_{k}|a\rangle+b_{k}|b\rangle+c_{k}|c\rangle$ being the bulk state and

$$
H(k)=\left(\begin{array}{ccc}
-i \gamma_{a} & g_{r} & g_{l} e^{-i k} \\
g_{r} & \delta_{0} & g_{0} \\
g_{l} e^{i k} & g_{0} & -\delta_{0}
\end{array}\right)
$$

being the bulk Hamiltonian of eigenvalue $E(k)$. In the momentum space, it is convenient to diagonalize the subspace of the neutral sites to attain

$$
H^{\prime}(k)=\left(\begin{array}{ccc}
-i \gamma_{a} & g_{+} & g_{-} \\
g_{+}^{*} & \delta_{+} & 0 \\
g_{-}^{*} & 0 & \delta_{-}
\end{array}\right)
$$

with $\delta_{ \pm}= \pm \sqrt{g_{0}^{2}+\delta_{0}^{2}}$ being the effective on-site potentials (detunings) and

$$
g_{ \pm}=\frac{g_{0} g_{r}-\left(\delta_{0} \mp \sqrt{g_{0}^{2}+\delta_{0}^{2}}\right) g_{l} e^{-i k}}{\sqrt{2\left(g_{0}^{2}+\delta_{0}^{2} \mp \delta_{0} \sqrt{g_{0}^{2}+\delta_{0}^{2}}\right)}}
$$

being the effective couplings. Then the bulk state can be rewritten as $\left|\psi_{k}^{\prime}\right\rangle=a_{k}|a\rangle+b_{k}^{+}\left|b_{+}\right\rangle+b_{k}^{-}\left|b_{-}\right\rangle$with

$$
\left|b_{ \pm}\right\rangle=\frac{g_{0}|b\rangle-\left(\delta_{0} \mp \sqrt{g_{0}^{2}+\delta_{0}^{2}}\right)|c\rangle}{\sqrt{2\left(g_{0}^{2}+\delta_{0}^{2} \mp \delta_{0} \sqrt{g_{0}^{2}+\delta_{0}^{2}}\right)}} .
$$

According to Eq. (7), we can find that

(i) $g_{+}=0 \quad$ when both $k=\pi$ and $\frac{g_{l}}{g_{r}}=\frac{g_{0}}{\sqrt{g_{0}^{2}+\delta_{0}^{2}}-\delta_{0}}$;

(ii) $g_{-}=0 \quad$ when both $k=0$ and $\frac{g_{l}}{g_{r}}=\frac{g_{0}}{\sqrt{g_{0}^{2}+\delta_{0}^{2}}+\delta_{0}}$.
In case (i), $\left|b_{+}\right\rangle$is decoupled from both $|a\rangle$ and $\left|b_{-}\right\rangle$so that it becomes a dark state immune to the lossy sites [25,27,28,37]. In this case, we have

$$
\left.\delta g_{+} \equiv \delta g\right|_{g_{+}=0}=\frac{g_{0}+\delta_{0}-\sqrt{g_{0}^{2}+\delta_{0}^{2}}}{g_{0}-\delta_{0}+\sqrt{g_{0}^{2}+\delta_{0}^{2}}} .
$$

Similarly, $\left|b_{-}\right\rangle$is a dark state in case (ii), with

$$
\left.\delta g_{-} \equiv \delta g\right|_{g_{-}=0}=\frac{g_{0}-\delta_{0}-\sqrt{g_{0}^{2}+\delta_{0}^{2}}}{g_{0}+\delta_{0}+\sqrt{g_{0}^{2}+\delta_{0}^{2}}} .
$$

It is clear that the two dark states $\left|b_{ \pm}\right\rangle$corresponding to $\delta g_{ \pm}$ are simultaneously attained only for $\delta_{0}=0$. Importantly, we find that $\delta g_{-}\left(\delta g_{+}\right)$exactly predicts the center of the left (right) topological phase transition (see vertical lines in Fig. 2). Therefore, we may conclude that each topological transition indicates the appearance of a dark state in the $3 \times 3$ Hilbert space as the coupling imbalance $\delta g$ is scanned. Moreover, we reveal that there are three nondegenerate dark states for a tetramerized lattice containing only one lossy site in each unit cell (see the Appendix for details).

\section{Zak phase}

The two-step topological phase transition can be further understood by examining the Zak phase of Hamiltonian $H^{\prime}(k)$, which owns the right eigenvectors

$$
\left|\psi_{k, \alpha}^{\prime}\right\rangle=\frac{1}{\sqrt{\eta_{\alpha}}}\left(1, \frac{g_{+}^{*}}{\lambda_{\alpha}-\delta_{+}}, \frac{g_{-}^{*}}{\lambda_{\alpha}-\delta_{-}}\right)^{T},
$$

except when our model suffers a dark state. Here, $1 / \sqrt{\eta_{\alpha}}$ is a normalization factor and $\alpha \in\{1,2,3\}$ labels the three eigenvalues $\lambda$ which satisfy

$$
\begin{aligned}
& \lambda^{3}+\left(i \gamma_{a}-\delta_{+}-\delta_{-}\right) \lambda^{2}+\left(\delta_{+} \delta_{-}-i \gamma_{a} \delta_{+}-i \gamma_{a} \delta_{-}\right) \lambda \\
& \quad-\left(\left|g_{+}\right|^{2}+\left|g_{-}\right|^{2}\right) \lambda+i \gamma_{a} \delta_{+} \delta_{-}+\left|g_{+}\right|^{2} \delta_{-}+\left|g_{-}\right|^{2} \delta_{+}=0 .
\end{aligned}
$$

Since $\left[H^{\prime}\left(k, \gamma_{a}\right)\right]^{\dagger}=H^{\prime}\left(k,-\gamma_{a}\right)$ and $\left[\lambda\left(k, \gamma_{a}\right)\right]^{*}=\lambda\left(k,-\gamma_{a}\right)$, the left eigenvectors of $H^{\prime}(k)$ can be easily attained through $\left|\varphi_{k, \alpha}^{\prime}\left(\gamma_{a}\right)\right\rangle=\left|\psi_{k, \alpha}^{\prime}\left(-\gamma_{a}\right)\right\rangle$ as

$$
\left\langle\varphi_{k, \alpha}^{\prime}\right|=\frac{1}{\sqrt{\eta_{\alpha}}}\left(1, \frac{g_{+}}{\lambda_{\alpha}-\delta_{+}}, \frac{g_{-}}{\lambda_{\alpha}-\delta_{-}}\right) .
$$

The normalization factor $1 / \sqrt{\eta_{\alpha}}$ is chosen to ensure the biorthogonal relation $\left\langle\varphi_{k, \alpha}^{\prime} \mid \psi_{k, \beta}^{\prime}\right\rangle=\delta_{\alpha, \beta}$ by setting

$$
\eta_{\alpha}=1+\frac{\left|g_{+}\right|^{2}}{\left(\lambda_{\alpha}-\delta_{+}\right)^{2}}+\frac{\left|g_{-}\right|^{2}}{\left(\lambda_{\alpha}-\delta_{-}\right)^{2}}
$$

With the left and right eigenvectors, we can define the Zak phase for individual energy bands as

$$
z_{\alpha}=i \int_{-\pi}^{\pi}\left\langle\varphi_{k, \alpha}^{\prime} \mid \partial_{k} \psi_{k, \alpha}^{\prime}\right\rangle d k
$$

except at the phase transition points. Substituting Eqs. (9) and (10) into Eq. (11), we obtain

$$
z_{\alpha}=\int_{-\pi}^{\pi} \frac{i d k}{2 \eta_{\alpha}}\left[\frac{f_{+}}{\left(\lambda_{\alpha}-\delta_{+}\right)^{2}}+\frac{f_{-}}{\left(\lambda_{\alpha}-\delta_{-}\right)^{2}}\right]
$$




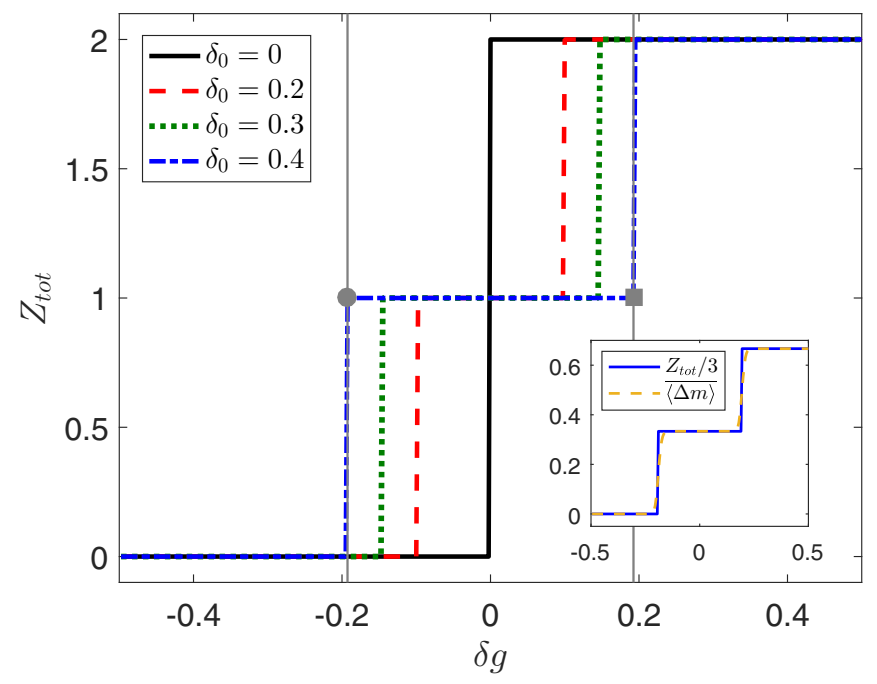

FIG. 3. Total Zak phase $Z_{\text {tot }}$ against coupling imbalance $\delta g$ for different values of $\delta_{0}$. The gray square and dot correspond to $\delta g_{ \pm}$ in the case of $\delta_{0}=0.4$, respectively. The inset shows $Z_{\text {tot }} / 3$ and the globally averaged displacement $\overline{\langle\Delta m\rangle}$ against $\delta g$ for $\delta_{0}=0.4$. Other parameters are the same as in Fig. 2.

with $f_{ \pm}=g_{ \pm} \partial_{k} g_{ \pm}^{*}-g_{ \pm}^{*} \partial_{k} g_{ \pm}$.

Note in the absence of chiral symmetry, the individualband Zak phases are usually not quantized [46]. However, the total Zak phase in units of $2 \pi$,

$$
Z_{\mathrm{tot}}=-\frac{1}{2 \pi} \sum_{\alpha} z_{\alpha}
$$

is always an integer and thus can be used to characterize the topological phases of 1D TIs [65].

We plot in Fig. 3 the total Zak phase $Z_{\text {tot }}$ as a function of $\delta g$ for four typical values of $\delta_{0}$. Clearly, it shows an abrupt single-phase transition in the case of $\delta_{0}=0$ and changes from 0 to 2 through an intermediate value 1 as long as $\delta_{0} \neq 0$. The positions of the phase transitions are closely related to the dark states above and are exactly predicted by $\delta g_{ \pm}$(see, e.g., gray dot and square in the case of $\delta_{0}=0.4$ ). It is worth pointing out that denoting by $\overline{\langle\Delta m\rangle}$ the global average of $\langle\Delta m\rangle$ over three independent initial states (i.e., $|0, a\rangle,|0, b\rangle$, and $|0, c\rangle$ ), the relation anticipated in Ref. [28] $Z_{\mathrm{tot}}=p \overline{\langle\Delta m\rangle}$ with $p$ being the number of sites per unit cell is indeed verified. This can be seen from the inset in Fig. 3, where the value of $\overline{\langle\Delta m\rangle}$ as well as the positions of phase transitions are in good agreement with those of $Z_{\text {tot }} / 3$. The same happens for the tetramerized lattice considered in the Appendix with $p=4$.

\section{ENERGY SPECTRA AND MIDGAP EDGE STATES}

In non-Hermitian TIs, the conventional bulk-boundary correspondence may be invalid while the real-space energy spectra are always reliable in predicting the emergence of topological edge states $[13,45]$. In view of this, we plot in Fig. 4 the real-space energy spectra of the finite sample considered in Fig. 2. As shown in Figs. 4(a) and 4(b), in the case of $\delta_{0}=0$, two midgap states of $\operatorname{Re}(E)= \pm 1$ and $\operatorname{Im}(E)=0$ emerge within two symmetric band gaps for $\delta g>0$, indicating a single topological transition around $\delta g=0$ from the trivial
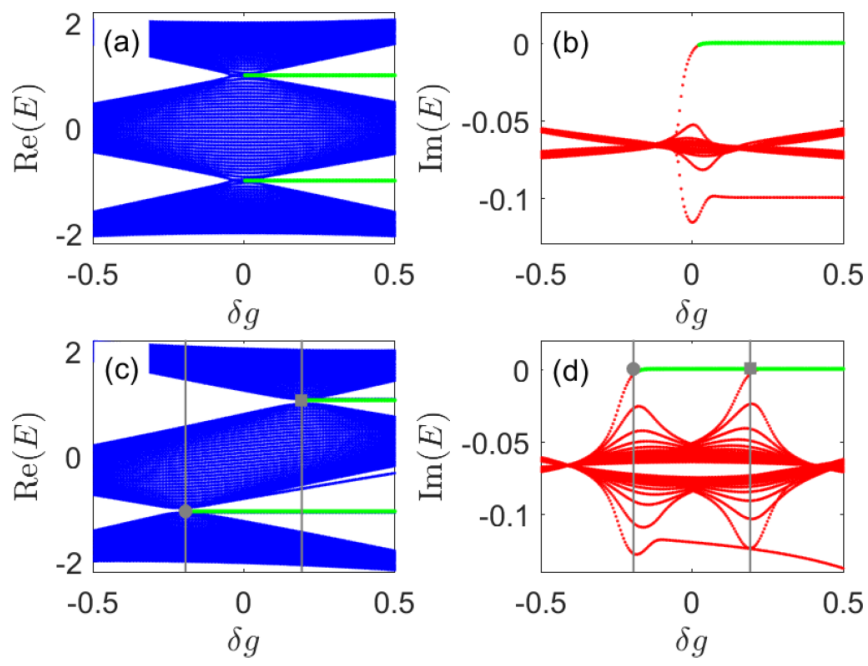

FIG. 4. Real (a, c) and imaginary (b, d) energy spectra of $H(m)$ against coupling imbalance $\delta g$ for $\delta_{0}=0(\mathrm{a}, \mathrm{b})$ and $\delta_{0}=0.4(\mathrm{c}$, d). In each panel, the topological edge modes are marked in green. The gray square and dot correspond to $\delta g_{ \pm}$in the case of $\delta_{0}=0.4$, respectively. Other parameters are the same as in Fig. 2.

phase with no midgap state to the nontrivial phase with two midgap states. It is worth noting that only the two midgap states are stable (the imaginary parts of eigenvalues are zero) while all other states will vanish with time (the imaginary parts of eigenvalues are negative). That is to say, the two midgap states are in fact the dark states mentioned above. In the case of $\delta_{0}=0.4$, however, we find that the upper and lower band gaps are markedly asymmetric so that only one midgap state emerges for $-0.1926<\delta g<0.1926$ while two midgap states emerge for $\delta g>0.1926$, as shown in Figs. 4(c) and 4(d). This indicates two topological transitions around $\delta g= \pm 0.1926$ with distinct topological phases characterized by different numbers of midgap states. Note that in Fig. 4(c),
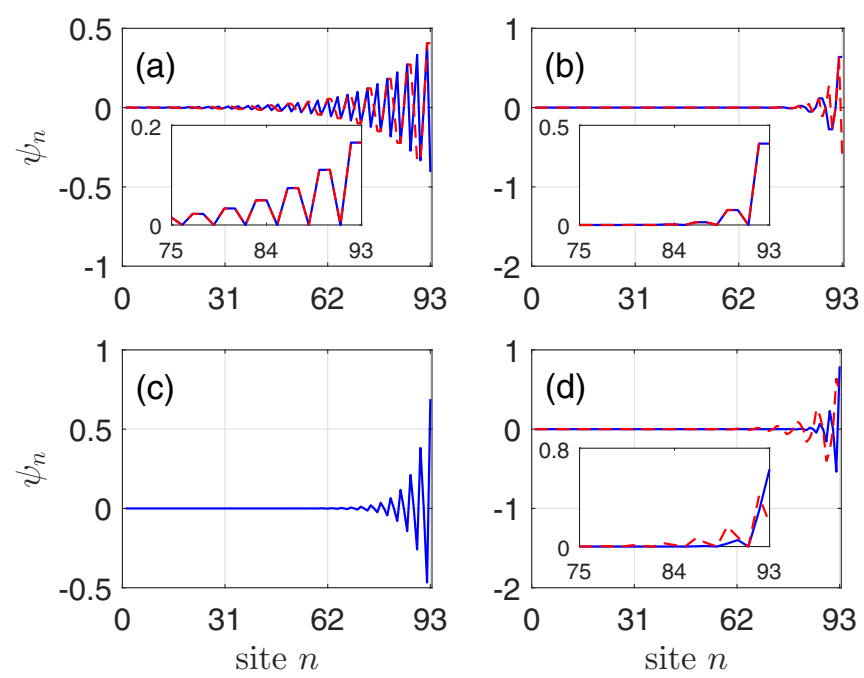

FIG. 5. Real-space wave functions of the midgap edge states with $\delta_{0}=0, \delta g=0.1$ (a); $\delta_{0}=0, \delta g=0.4$ (b); $\delta_{0}=0.4, \delta g=0.1$ (c); $\delta_{0}=0.4, \delta g=0.4$ (d). The insets depict the wave-function probability profiles $\left|\psi_{n}\right|^{2}$. Other parameters are the same as in Fig. 2. 


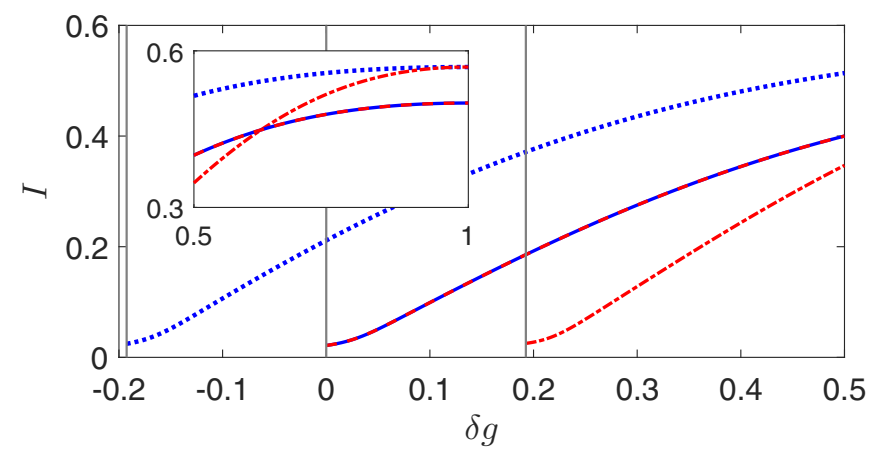

FIG. 6. Inverse participation ratios of the midgap edge states against coupling imbalance $\delta g$. The red dashed (dot-dashed) and blue solid (dotted) lines correspond to the midgap states in the upper and lower band gaps in the case of $\delta_{0}=0\left(\delta_{0}=0.4\right)$, respectively. The three vertical lines denote the emergence positions of corresponding midgap states. Other parameters are the same as in Fig. 2.

an additional state can be found, the eigenvalue of which has a real part that splits from the band for large enough $\delta g$ : this state is localized at the left boundary of the lattice and suffers strong losses. We do not consider it further, however, as it is not dark and thereby not connected to the topological invariants. More importantly, the two band closing points are consistent with the phase transition points predicted by $\delta g_{ \pm}$ (see the gray square and dot), implying that the bulk-boundary correspondence remains valid in our model.

For a deeper insight, we also plot in Fig. 5 the real-space wave functions $\psi_{n}$ of the midgap states, being $\psi_{n}$ equal to $a_{m}, b_{m}$, and $c_{m}$ when $n=3 m-2, n=3 m-1$, and $n=3 m$, respectively. As shown in Figs. 5(a) and 5(b), in the case of $\delta_{0}=0$, there are always two edge states (i.e., the two midgap states) localized at the right boundary as long as $\delta g>0$, which become more and more localized as $\delta g$ is increased. The wave functions of the two midgap edge states exhibit different phases and identical modulus (see probability profiles $\left|\psi_{n}\right|^{2}$ in the insets). The same calculations are made in Figs. 5(c) and 5(d) for the midgap states in the case of $\delta_{0}=0.4$ to gain a direct comparison. It is clear that, as predicted by the real-space energy spectra, we observe only one edge state for $\delta g_{-}<\delta g<\delta g_{+}$while two edge states for $\delta g>\delta g_{+}$at the right boundary. More interestingly, the probability profiles of the two edge states are distinguishable in this case, i.e., the moduli of the wave functions become different.

To quantify the localization degrees of the edge states, we then introduce the inverse participation ratios (IPRs) [66,67]

$$
I=\frac{\sum_{n}\left|\psi_{n}\right|^{4}}{\left(\sum_{n}\left|\psi_{n}\right|^{2}\right)^{2}}
$$

with a larger value indicating a stronger localization. As in other 1D systems, the IPRs of the bulk states roughly equal the inverse of the lattice size, i.e., $I \simeq 1 / 3(2 N+1)$ in our system. The edge states, however, typically exhibit much larger IPRs depending, e.g., on $\delta g$ and $\delta_{0}$ as shown in Fig. 6. In the case of $\delta_{0}=0$, the two midgap edge states exhibit identical probability profiles $\left|\psi_{n}\right|^{2}$ as discussed above, so their IPRs are also the same though increasing monotonically with $\delta g$. In the case of $\delta_{0}=0.4$, the midgap state in the lower (upper) band gap is more localized (extended) with a larger (smaller) IPR as compared to the two midgap states attained with $\delta_{0}=0$. We further note from the inset that in this case, the IPR of the upper midgap state grows more rapidly than, and finally approaches, that of the lower midgap state as we increase $\delta g$.

Finally, we point out that the midgap edge states are topologically protected in spite of the absence of unitary and/or
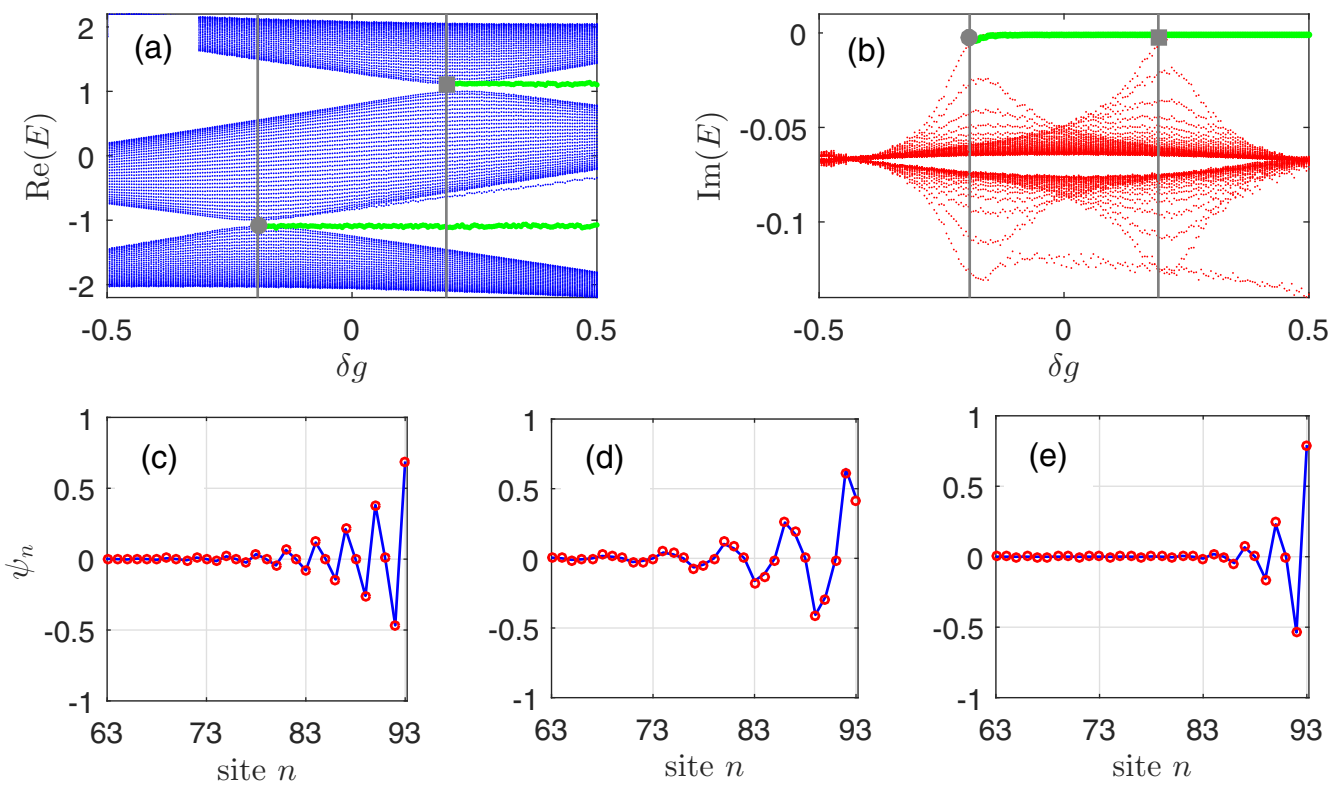

FIG. 7. Real (a) and imaginary (b) energy spectra of $H(m)$ against coupling imbalance $\delta g$ in the presence of disorders with $\left\{\rho_{\beta, m}, \Delta_{m}\right\} \in$ $[-0.05,0.05]$. The gray square and circle correspond to $\delta g_{ \pm}$in the case of $\delta_{0}=0.4$, respectively. Real-space wave functions of the lower midgap states with $\delta g=0.1$ (c), the upper (d) and the lower (e) midgap states with $\delta g=0.4$. The blue lines and red circles correspond to the cases without and with disorders, respectively. Other parameters are the same as in Fig. 2. 
antiunitary symmetries in our model. The topological protection here is associated with the dark states decoupled from all lossy sites [25,27,28], and can be proved by introducing disorders in both coupling constants and on-site potentials, i.e., $g_{\beta} \rightarrow g_{\beta}+\rho_{\beta, m}(\beta=0, l, r)$ and $\delta_{0} \rightarrow \delta_{0}+\Delta_{m}$ with $-0.05 \leqslant\left\{\rho_{\beta, m}, \Delta_{m}\right\} \leqslant 0.05$ being four sets of independent real random numbers. We can find from Figs. 7(a) and 7(b) that both midgap states persist within the corresponding band gaps and remain stable (i.e., the imaginary parts of their eigenvalues remain vanishing) even in the presence of all types of disorders. The two staggered topological transition points are insensitive to the disorders as well, so they still can be predicted by $\delta g_{ \pm}$. We also illustrate in Figs. 7(c) and 7(d) the wave functions of relevant midgap states attained with (red circles) and without (blue lines) disorders. The highly coincident wave functions further verify that the midgap states in our model are robust against disorders as those in symmetry-protected TIs.

\section{CONCLUSIONS}

In summary, we have studied the nontrivial topological features of a 1D lossy trimerized lattice by focusing on two topological invariants, i.e., the mean displacement $\langle\Delta m\rangle$ and the total Zak phase. It is found that a two-step phase transition of quantum transport could occur between $\langle\Delta m\rangle=$ 0 and $\langle\Delta m\rangle=1$ through a fractional number, whose value and extension can be easily tuned by modulating the on-site potentials of neutral sites and the initial state of lattice excitation. We have also revealed that each topological transition corresponds to a dark state decoupled from the lossy sites, associated with one band closing point, and is accompanied by a change in the midgap-state number (and also $Z_{\text {tot }}$, the total Zak phase in units of $2 \pi$ ) from 0 to 1 or from 1 to 2. The midgap edge states are, in particular, topologically protected (robust against disorders) with their localization degrees mainly controlled by the coupling imbalance $\delta g$. Finally, It is shown in the Appendix that the results in this paper can also be extended to a lossy tetramerized model which exhibits a three-step topological transition.

\section{ACKNOWLEDGMENTS}

This work is supported by the National Natural Science Foundation of China (No. 10534002 and No. 11674049); the Cooperative Program by the Italian Ministry of Foreign Affairs and International Cooperation (No. PGR00960) and the National Natural Science Foundation of China (No. 11861131001).

\section{APPENDIX: EXTENSION TO A TETRAMERIZED LATTICE}

Here we extend our discussion to a tetramerized lattice consisting of one lossy site $\left(A_{m}\right)$ and three neutral sites $\left(B_{m}\right.$, $C_{m}$, and $\left.D_{m}\right)$ in each unit cell. In this extended model, the intercell (intracell) coupling constant between sites $D_{m-1}\left(A_{m}\right)$ and $A_{m}\left(B_{m}\right)$ is denoted by $g_{l}\left(g_{r}\right)$, while the intracell coupling constant between sites $B_{m}\left(C_{m}\right)$ and $C_{m}\left(D_{m}\right)$ is denoted by $g_{c}$. Note that, similar to the trimerized lattice considered in the main text, this system lacks chiral and inversion symmetry, but it is a nearest-neighbor lattice with only one lossy site per unit cell, and thus it is also expected to have a topologically protected quantum transport [28]. By considering the periodic boundary condition, the bulk Hamiltonian can be given by

$$
H(k)=\left(\begin{array}{cccc}
-i \gamma & g_{r} & 0 & g_{l} e^{-i k} \\
g_{r} & \delta & g_{c} & 0 \\
0 & g_{c} & 0 & g_{c} \\
g_{l} e^{i k} & 0 & g_{c} & -\delta
\end{array}\right),
$$

where we have assumed $\gamma_{a}=\gamma, \delta_{b}=\delta, \delta_{c}=0$, and $\delta_{d}=-\delta$ for simplicity. Likewise, we assume here $g_{l}=g_{c}+\delta g$ and $g_{r}=g_{c}-\delta g$ with $\delta g$ being the coupling imbalance. To seek appropriate situations under which dark states can be realized, we now diagonalize the subspace of the three neutral sites to obtain an effective bulk Hamiltonian, i.e.,

$$
H^{\prime}(k)=\left(\begin{array}{cccc}
-i \gamma & g_{+} & g_{0} & g_{-} \\
g_{+}^{*} & \delta_{+} & 0 & 0 \\
g_{0} & 0 & \delta_{0} & 0 \\
g_{-}^{*} & 0 & 0 & \delta_{-}
\end{array}\right),
$$

with three effective on-site potentials $\delta_{0}=0$ and $\delta_{ \pm}=$ $\pm \sqrt{2 g_{c}^{2}+\delta^{2}}$, and three effective coupling constants

$$
\begin{aligned}
g_{0} & =\frac{g_{c} g_{r}-g_{c} g_{l} e^{-i k}}{\sqrt{2 g_{c}^{2}+\delta^{2}}}, \\
g_{ \pm} & =\frac{g_{r}\left(\delta \pm \sqrt{2 g_{c}^{2}+\delta^{2}}\right)-g_{l}\left(\delta \mp \sqrt{2 g_{c}^{2}+\delta^{2}}\right) e^{-i k}}{2 \sqrt{2 g_{c}^{2}+\delta^{2}}} .
\end{aligned}
$$

In this case, we have the new bulk state $\left|\psi_{k}\right\rangle=a_{k}|a\rangle+$ $b_{k}^{+}\left|b_{+}\right\rangle+b_{k}^{0}\left|b_{0}\right\rangle+b_{k}^{-}\left|b_{-}\right\rangle$with

$$
\begin{aligned}
\left|b_{0}\right\rangle & =\frac{g_{c}|b\rangle-\delta|c\rangle-g_{c}|d\rangle}{\sqrt{2 g_{c}^{2}+\delta^{2}}}, \\
\left|b_{ \pm}\right\rangle & =\frac{\delta|b\rangle+2 g_{c}|c\rangle-\delta|d\rangle}{2 \sqrt{2 g_{c}^{2}+\delta^{2}}} \pm \frac{|b\rangle+|d\rangle}{2} .
\end{aligned}
$$

We can see clearly from Eq. (A3) that (i) $g_{0}=0$ when $k=0$ and $g_{l} / g_{r}=1 \quad\left(\delta g_{0}=\left.\delta g\right|_{g_{0}=0}=0\right)$ such that $\left|b_{0}\right\rangle$ becomes a dark state; and (ii) $g_{ \pm}=0$ when $k=$ $\pi$ and $g_{l} / g_{r}=\left(\sqrt{2 g_{c}^{2}+\delta^{2}} \pm \delta\right) /\left(\sqrt{2 g_{c}^{2}+\delta^{2}} \mp \delta\right) \quad\left(\delta g_{ \pm}=\right.$ $\left.\left.\delta g\right|_{g_{ \pm}=0}= \pm \delta / \sqrt{2 g_{c}^{2}+\delta^{2}}\right)$ such that $\left|b_{ \pm}\right\rangle$become two other nondegenerate dark states.

Accordingly, we expect a three-step staggered topological transition from $\langle\Delta m\rangle=0$ to $\langle\Delta m\rangle=1$ through two different fractional values as the coupling imbalance $\delta g$ is varied. Figure 8 (a) shows the globally averaged displacement $\overline{\langle\Delta m\rangle}$ as a function of $\delta g$ in the case of $\delta=0.4$, which does exhibit three phase transitions with the center of each one exactly predicted by $\delta g_{ \pm}$and $\delta g_{0}$, respectively. Moreover, we can find from Figs. $8(\mathrm{~b})$ and $8(\mathrm{c})$ that each phase transition is accompanied by the emergence or disappearance of a new midgap state and all of them are stable in this model as well. As shown in Figs. 8(d)-8(f), all midgap states are localized at the right boundary and their number changes from 0 to 1 to 2 to 3 as $\delta g$ increases across the four distinct topological phases. Once again, the midgap edge states exhibit different localization degrees, which can be significantly enhanced by increasing 

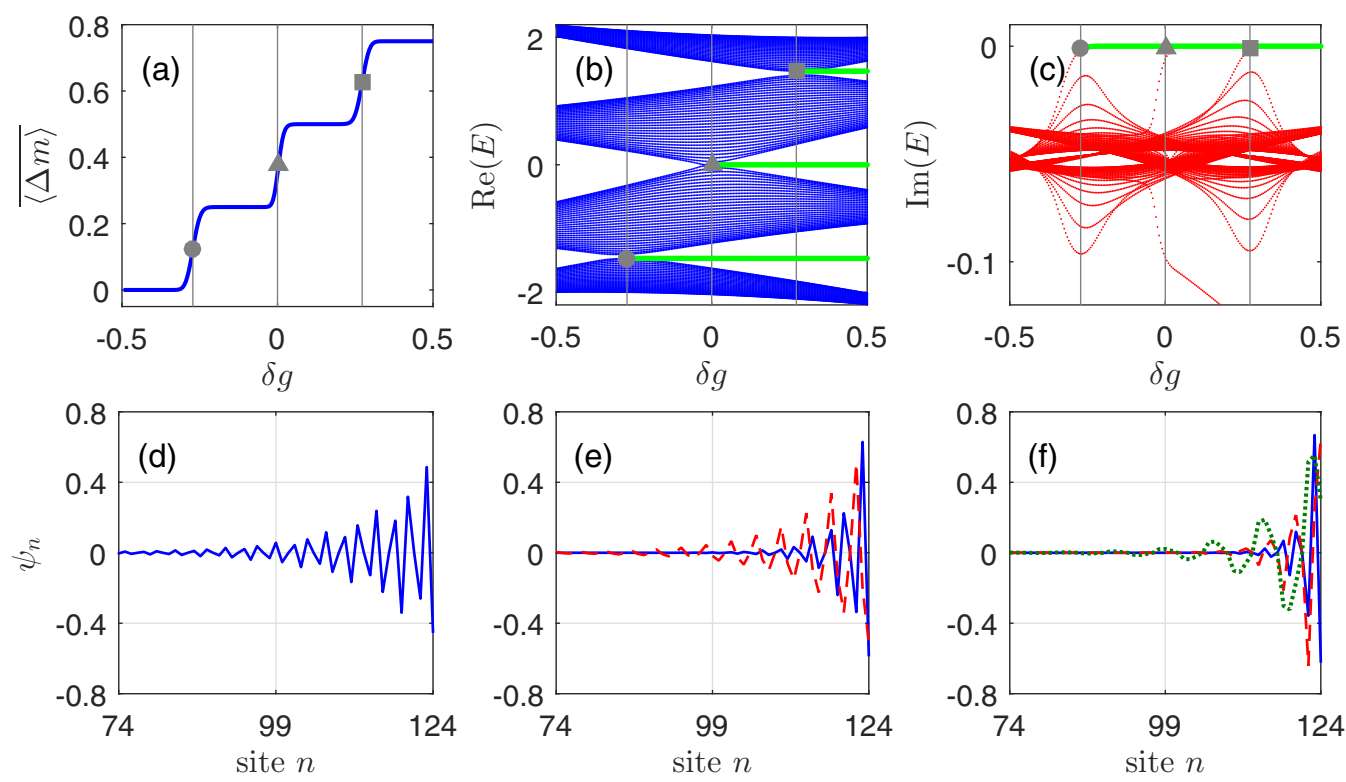

FIG. 8. Globally averaged displacement $\overline{\langle\Delta m\rangle}$ (a), and real (b) and imaginary (c) energy spectra of the tetramerized model against coupling imbalance $\delta g$ with $\delta=0.4$. The gray square, circle, and triangle correspond to $\delta g_{ \pm}$and $\delta g_{0}$ in this case, respectively. Real-space wave functions of the midgap edge states with $\delta g=-0.1(\mathrm{~d}), \delta g=0.2(\mathrm{e})$, and $\delta g=0.5$ (f). The blue solid, red dashed, and green dotted lines correspond to the midgap states in the lower, middle, and upper band gaps, respectively. Other parameters are $\gamma=0.2$ and $N=15$.

$\delta g$. More generally, we note that a 1D nearest-neighbor lattice containing $p$ sites per unit cell of which only one is lossy can be classified as a weak-bipartite system [28]. One may expect in such a model a $(p-1)$-step quantized quantum transport with $(p-2)$ intermediate phases characterized by different fractional mean displacements, and at most $(p-1)$ nondegenerate dark states (topologically protected midgap states).
[1] M. Z. Hasan and C. L. Kane, Colloquium: Topological insulators, Rev. Mod. Phys. 82, 3045 (2010).

[2] X.-L. Qi and S.-C. Zhang, Topological insulators and superconductors, Rev. Mod. Phys. 83, 1057 (2011).

[3] Y. C. Hu and T. L. Hughes, Absence of topological insulator phases in non-Hermitian $\mathcal{P} \mathcal{T}$-symmetric Hamiltonians, Phys. Rev. B 84, 153101 (2011).

[4] K. Esaki, M. Sato, K. Hasebe, and M. Kohmoto, Edge states and topological phases in non-Hermitian systems, Phys. Rev. B 84, 205128 (2011).

[5] H. Schomerus, Topologically protected midgap states in complex photonic lattices, Opt. Lett. 38, 1912 (2014).

[6] S. Malzard, C. Poli, and H. Schomerus, Topologically Protected Defect States in Open Photonic Systems with Non-Hermitian Charge-Conjugation and Parity-Time Symmetry, Phys. Rev. Lett. 115, 200402 (2015).

[7] C. Yuce, Topological phase in a non-Hermitian $\mathcal{P} \mathcal{T}$ symmetric system, Phys. Lett. A 379, 1213 (2015).

[8] T. E. Lee, Anomalous Edge State in a Non-Hermitian Lattice, Phys. Rev. Lett. 116, 133903 (2016).

[9] D. Leykam, K. Y. Bliokh, C. Huang, Y. D. Chong, and F. Nori, Edge Modes, Degeneracies, and Topological Numbers in NonHermitian Systems, Phys. Rev. Lett. 118, 040401 (2017).

[10] S. Weimann, M. Kremer, Y. Plotnik, Y. Lumer, S. Nolte, K. G. Makris, M. Segev, M. C. Rechtsman, and A. Szameit, Topologically protected bound states in photonic parity-time-symmetric crystals, Nat. Mater. 16, 433 (2017).
[11] Y. Xu, S.-T. Wang, and L.-M. Duan, Weyl Exceptional Rings in a Three-Dimensional Dissipative Cold Atomic Gas, Phys. Rev. Lett. 118, 045701 (2017).

[12] F. K. Kunst, E. Edvardsson, J. C. Budich, and E. J. Bergholtz, Biorthogonal Bulk-Boundary Correspondence in Non-Hermitian Systems, Phys. Rev. Lett. 121, 026808 (2018).

[13] Z. Gong, Y. Ashida, K. Kawabata, K. Takasan, S. Higashikawa, and M. Ueda, Topological Phases of Non-Hermitian Systems, Phys. Rev. X 8, 031079 (2018).

[14] S. Longhi, Topological Phase Transition in NonHermitian Quasicrystals, Phys. Rev. Lett. 122, 237601 (2019).

[15] L. Lu, J. D. Joannopoulos, and M. Soljačić, Topological Photonics, Nat. Photon. 8, 821 (2014).

[16] B. Midya, H. Zhao, and L. Feng, Non-Hermitian photonics promises exceptional topology of light, Nat. Commun. 9, 2674 (2018).

[17] T. Ozawa, H. M. Price, A. Amo, N. Goldman, M. Hafezi, L. Lu, M. C. Rechtsman, D. Schuster, J. Simon, O. Zilberberg, and I. Carusotto, Topological photonics, Rev. Mod. Phys. 91, 015006 (2019).

[18] M. G. Silveirinha, Topological theory of non-Hermitian photonic systems, Phys. Rev. B 99, 125155 (2019).

[19] M. Hafezi, E. A. Demler, M. D. Lukin, and J. M. Taylor, Robust optical delay lines with topological protection, Nat. Phys. 7, 907 (2011). 
[20] Z. Wang, Y. Chong, J. D. Joannopoulos, and M. Soljačić, Observation of unidirectional backscattering-immune topological electromagnetic states, Nature 461, 772 (2009).

[21] T. Karzig, C.-E. Bardyn, N. H. Lindner, and G. Refael, Topological Polaritons, Phys. Rev. X 5, 031001 (2015).

[22] L. Pilozzi and C. Conti, Topological lasing in resonant photonic structures, Phys. Rev. B 93, 195317 (2016).

[23] P. St-Jean, V. Goblot, E. Galopin, A. Lemaitre, T. Ozawa, L. Le Gratiet, I. Sagnes, J. Bloch, and A. Amo, Lasing in topological edge states of a one-dimensional lattice, Nat. Photonics 11, 651 (2017).

[24] M. Parto, S. Wittek, H. Hodaei, G. Harari, M. A. Bandres, J. Ren, M. C. Rechtsman, M. Segev, D. N. Christodoulides, and M. Khajavikhan, Edge-Mode Lasing in 1D Topological Active Arrays, Phys. Rev. Lett. 120, 113901 (2018).

[25] M. S. Rudner and L. S. Levitov, Topological Transition in a Non-Hermitian Quantum Walk, Phys. Rev. Lett. 102, 065703 (2009).

[26] L.-H. Li, Z.-H. Xu, and S. Chen, Topological phases of generalized Su-Schrieffer-Heeger models, Phys. Rev. B 89, 085111 (2014).

[27] J. M. Zeuner, M. C. Rechtsman, Y. Plotnik, Y. Lumer, S. Nolte, M. S. Rudner, M. Segev, and A. Szameit, Observation of a Topological Transition in the Bulk of a Non-Hermitian System, Phys. Rev. Lett. 115, 040402 (2015).

[28] M. S. Rudner, M. Levin, and L. S. Levitov, Survival, decay, and topological protection in non-Hermitian quantum transport, arXiv:1605.07652v1.

[29] L. CamposVenuti, Z. Ma, H. Saleur, and S. Haas, Topological protection of coherence in a dissipative environment, Phys. Rev. A 96, 053858 (2017).

[30] C. Li, S. Lin, G. Zhang, and Z. Song, Topological nodal points in two coupled Su-Schrieffer-Heeger chains, Phys. Rev. B 96, 125418 (2017).

[31] S. Lieu, Topological phases in the non-Hermitian Su-SchriefferHeeger model, Phys. Rev. B 97, 045106 (2018).

[32] L.-J. Lang, Y. Wang, H. Wang, and Y. D. Chong, Effects of nonHermiticity on Su-Schrieffer-Heeger defect states, Phys. Rev. B 98, 094307 (2018).

[33] M. J. Kastoryano, and M. S. Rudner, Topological transport in the steady state of a quantum particle with dissipation, Phys. Rev. B 99, 125118 (2019).

[34] L. Du, J.-H. Wu, M. Artoni, and G. C. La Rocca, Phasedependent topological interface state and spatial adiabatic passage in a generalized Su-Schrieffer-Heeger model, Phys. Rev. A 100, 012112 (2019).

[35] S. Ganeshan, K. Sun, and S. DasSarma, Topological ZeroEnergy Modes in Gapless Commensurate Aubry-André-Harper Models, Phys. Rev. Lett. 110, 180403 (2013).

[36] A. K. Harter, T. E. Lee, and Y. N. Joglekar, $\mathcal{P} \mathcal{T}$-breaking threshold in spatially asymmetric Aubry-André and Harper models: Hidden symmetry and topological states, Phys. Rev. A 93, 062101 (2016).

[37] X. L. Zhao, Z. C. Shi, C. S. Yu, and X. X. Yi, Effect of loss on the topological features of dimer chains described by the extended Aubry-André-Harper model, Phys. Rev. A 95, 043837 (2017).

[38] X. H. Wang, T. T. Liu, Y. Xiong, and P. Q. Tong, Spontaneous $\mathcal{P} \mathcal{T}$-symmetry breaking in non-Hermitian Kitaev and extended Kitaev models, Phys. Rev. A 92, 012116 (2015).
[39] B.-G. Zhu, R. Lü, and S. Chen, $\mathcal{P} \mathcal{T}$ symmetry in the nonHermitian Su-Schrieffer-Heeger model with complex boundary potentials, Phys. Rev. A 89, 062102 (2014).

[40] M. Klett, H. Cartarius, D. Dast, J. Main, and G. Wunner, Relation between $\mathcal{P} \mathcal{T}$-symmetry breaking and topologically nontrivial phases in the Su-Schrieffer-Heeger and Kitaev models, Phys. Rev. A 95, 053626 (2017).

[41] C. Yuce, Edge states at the interface of non-Hermitian systems, Phys. Rev. A 97, 042118 (2018).

[42] H. Hatano and D. R. Nelson, Localization Transitions in Non-Hermitian Quantum Mechanics, Phys. Rev. Lett. 77, 570 (1996).

[43] S. Longhi, Non-Hermitian bidirectional robust transport, Phys. Rev. B 95, 014201 (2017).

[44] D. Leykam, S. Flach, and Y. D. Chong, Flat bands in lattices with non-Hermitian coupling, Phys. Rev. B 96, 064305 (2017).

[45] S. Yao and Z. Wang, Edge States and Topological in Variants of Non-Hermitian Systems, Phys. Rev. Lett. 121, 086803 (2018).

[46] J. Zak, Berry's Phase for Energy Bands in Solids, Phys. Rev. Lett. 62, 2747 (1989).

[47] M. Atala, M. Aidelsburger, J. T. Barreiro, D. Abanin, T. Kitagawa, E. Demler, and I. Bloch, Direct measurement of the Zak phase in topological Bloch bands, Nat. Phys. 9, 795 (2013).

[48] S. Longhi, Zak phase of photons in optical waveguide lattices, Opt. Lett. 38, 3716 (2013).

[49] L. Li, C. Yang, and S. Chen, Winding numbers of phase transition points for one-dimensional topological systems, Europhys. Lett. 112, 10004 (2015).

[50] J.-W. Rhim, J. Behrends, and J. H. Bardarson, Bulk-boundary correspondence from the intercellular Zak phase, Phys. Rev. B 95, 035421 (2017).

[51] L. Jin and Z. Song, Bulk-boundary correspondence in a nonHermitian system in one dimension with chiral inversion symmetry, Phys. Rev. B 99, 081103(R) (2019).

[52] C. Yin, H. Jiang, L. Li, R. Lü, and S. Chen, Geometrical meaning of winding number and its characterization of topological phases in one-dimensional chiral non-Hermitian systems, Phys. Rev. A 97, 052115 (2018).

[53] Y. Niu, S. B. Chung, C.-H. Hsu, I. Mandal, S. Raghu, and S. Chakravarty, Majorana zero modes in a quantum Ising chain with longer-ranged interactions, Phys. Rev. B 85, 035110 (2012).

[54] G. Zhang and Z. Song, Topological Characterization of Extended Quantum Ising Models, Phys. Rev. Lett. 115, 177204 (2015).

[55] F. Cardano, A. D’Errico, A. Dauphin, M. Maffei, B. Piccirillo, C. de Lisio, G. de Filippis, V. Cataudella, E. Santamato, L. Marrucci, M. Lewenstein, and P. Massignan, Detection of Zak phases and topological invariants in a chiral quantum walk of twisted photons, Nat. Commun. 8, 15516 (2017).

[56] T. Rakovszky, J. K. Asbóth, and A. Alberti, Detecting topological invariants in chiral symmetric insulators via losses, Phys. Rev. B 95, 201407(R) (2017).

[57] S. Longhi, Probing one-dimensional topological phases in waveguide lattices with broken chiral symmetry, Opt. Lett. 43, 4639 (2018).

[58] A. K. Harter, A. Saxena, and Y. N. Joglekar, Fragile aspects of topological transition in lossy and parity-time symmetric quantum walks, Sci. Rep. 8, 12065 (2018). 
[59] X.-Y. Xu, Q.-Q. Wang, W.-W. Pan, K. Sun, J.-S. Xu, G. Chen, J.-S. Tang, M. Gong, Y.-J. Han, C.-F. Li, and G.-C. Guo, Measuring the Winding Number in a Large-Scale Chiral Quantum Walk, Phys. Rev. Lett. 120, 260501 (2018).

[60] L. Xiao, X. Qiu, K. Wang, Z. Bian, X. Zhan, H. Obuse, B. C. Sanders, W. Yi, and P. Xue, Higher winding number in a nonunitary photonic quantum walk, Phys. Rev. A 98, 063847 (2018).

[61] V. M. Martinez Alvarez, and M. D. Coutinho-Filho, Edge states in trimer lattices, Phys. Rev. A 99, 013833 (2019).

[62] L. Jin, Topological phases and edge states in a non-Hermitian trimerized optical lattice, Phys. Rev. A 96, 032103 (2017).

[63] X. Liu and G. S. Agarwal, The new phases due to symmetry protected piecewise Berry phases; enhanced pump- ing and nonreciprocity in trimer lattices, Sci. Rep. 7, 45015 (2017).

[64] F. Minganti, A. Miranowicz, R. W. Chhajlany, and F. Nori, Quantum exceptional points of non-Hermitian Hamiltonians and Liouvillians: The effects of quantum jumps, arXiv: $1909.11619 \mathrm{v} 1$.

[65] S.-D. Liang and G.-Y. Huang, Topological invariance and global Berry phase in non-Hermitian systems, Phys. Rev. A 87, 012118 (2013).

[66] B. Kramer and A. MacKinnon, Localization: Theory and experiment, Rep. Prog. Phys. 56, 1469 (1993).

[67] F. Evers and A. D. Mirlin, Anderson transitions, Rev. Mod. Phys. 80, 1355 (2008). 